\title{
Optimization of Adaptive Controller Algorithm for a 6-DOF Robotic Manipulator
}

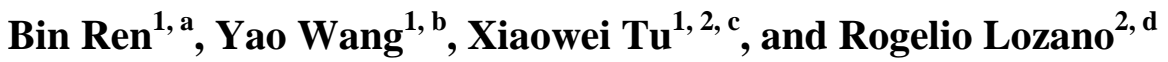 \\ ${ }^{1}$ School of Mechatronic Engineering and Automation,Shanghai University, Shanghai 200072, China \\ ${ }^{2}$ Université de Technologie de Compiègne, CNRS UMR 7253 Heudiasyc, France \\ abinren@i.shu.edu.cn, ${ }^{b}$ wangyao9504@126.com, ${ }^{c}$ tuxiaowei@shu.edu.cn, ${ }^{d}$ rogelio.lozano@hds.utc. \\ fr
}

Keywords: 6-DOF, adaptive controller algorithm, sliding mode surface

\begin{abstract}
Real time application of a 6-DOF robotic manipulator is very challenging and requires new robust control algorithms. When the unknown parameters of a robotic manipulator being changed, it is difficult to ensure the stability of the robotic manipulator system. Due to this phenomenon, an optimal adaptive algorithm is proposed for an existing 6-DOF robotic manipulator. This algorithm is based on the design of sliding mode surface to reduce the system position error, adding adaptive algorithm to compensate the instability of the system. The comparative simulation results prove that this algorithm has a good tracking performance, which can effectively eliminate the position steady-state error.
\end{abstract}

\section{Introduction}

The robotic manipulator technology is a comprehensive frontier discipline. Along with the progress of technology, the development of robotic manipulator has achieved fruitful results, and its application has contributed in many aspects. The traditional method of controlling the robotic manipulator is mainly PID control, which has the advantage that the control law is simple, easy to operate and does not require the model on the manipulator. However, due to the structure of the robotic manipulator is becoming more and more complex, and it has the characteristics such as strong coupling, nonlinearity and parameters changing, the conventional single control algorithm PID control algorithm cannot obtain satisfactory results.

For the above situation, domestic and foreign experts have put forward many solutions ${ }^{[1-4]}$. In [5], based on the expected compensation adaptive robust control algorithm, the integral parameter estimation of adaptive robust control is adjusted to the proportional integral parameter estimation and the accurate identification parameters are obtained by using the method of maximum likelihood parameter estimation. The simulation results show that the algorithm has significantly improved the stability and control-ability of the system. However, the trajectory tracking error of this algorithm is larger. In [6], it provides an adaptive control system for neural network error and the switching control gain of sliding mode control is adjusted by neural network. The simulation results show that 
this algorithm performs the automatic tracking with modeling error and uncertain interference, and improves the stability of the system. However, the algorithm has high accuracy in measuring position and velocity. In [7], it combines the PD control algorithm with the feed forward control algorithm and the simulation results show that this algorithm successfully reduces the average absolute error of the robotic manipulator. However, the algorithm lacks the stability analysis of the parameters of the controller. In [8], it combines the terminal sliding mode control with a boundary layer and the adaptive approach and the simulation results show that the algorithm can estimate this bound online and can assure a good performance. However, in the first 2 seconds, the torque of the mechanical arm fluctuates greatly.

In this paper, we will present different solutions to control an existing 6-DOF series robotic manipulator for research purpose. We first analyze the structure of the robotic manipulator, and use D-H method to construct the transformation matrix between each joint of the robotic manipulator, and deduce the positive kinematics model of the robotic manipulator.

Then, based on the traditional PD algorithm, we combine with the adaptive algorithm and sliding mode control algorithm. We design the sliding mode surface to eliminate the position error of the robotic manipulator, and add the adaptive controller to compensate the instability of the system. The simulation results show that the algorithm reduces the position error of the system and improves the estimated accuracy of unknown parameters and the position tracking performance of the robotic manipulator.

\section{Kinematics Model}

The 6-DOF series robotic manipulator mainly consists of a pedestal, a waist, an arm, a small arm, a wrist and a hand claw. Each part is connected by revolute joint and one joint formed one rotational degree of freedom. The mechanical manipulator is shown in Fig.1.

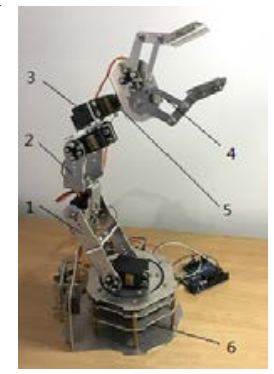

Fig.1 6-DOF robotic manipulator architecture model

1-waist, 2-arm , 3-small arm, 4-hand claw, 5-wrist , 6-pedestal

The motion analysis of the series robotic manipulator is to solve the position and posture of the hand claw knowing the motion parameters of each joint. In order to describe the movement of the robotic manipulator of the link, we usually use D-H (Denavit-Hartenberg) method to establish the coordinate system $^{[9]}$.

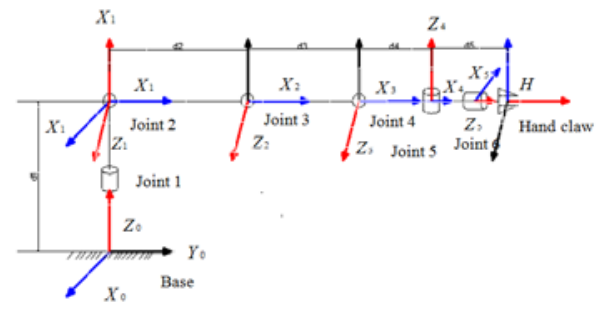

Fig.2 The coordinate system of links 
In Fig.2, we set up the coordinate system of links $\{i\}(i=1,2, \ldots, 6)$, let ${ }^{i-1} T_{i}$ as the transformation of links of coordinate system $\{i\}$ relative to $\{i-1\}$. The transformation of links ${ }^{i-1} T_{i}$ can be regarded as the coordinate system $\{i\}$ is obtained though the following four transformation:

1) revolved angle $\left(\alpha_{i-1}\right)$ around the axis $\left(x_{i-1}\right)$;

2) moved length $\left(a_{i-1}\right)$ along the axis $\left(x_{i-1}\right)$;

3) revolved angle $\left(\theta_{i}\right)$ around the axis $\left(z_{i}\right)$;

4) moved length $\left(d_{i}\right)$ along the axis $\left(z_{i}\right)$.

Then:

$$
{ }^{i-1} T_{i}=\operatorname{Rot}\left(x, \alpha_{i-1}\right) \operatorname{Trans}\left(x, a_{i-1}\right) \operatorname{Rot}\left(z, \theta_{i}\right) \operatorname{Trans}\left(z, d_{i}\right)
$$

Where

$$
\begin{aligned}
\operatorname{Rot}\left(x, \alpha_{i-1}\right) & =\left[\begin{array}{cccc}
1 & 0 & 0 & 0 \\
0 & c \alpha_{i-1} & -s \alpha_{i-1} & 0 \\
0 & s \alpha_{i-1} & c \alpha_{i-1} & 0 \\
0 & 0 & 0 & 1
\end{array}\right], \operatorname{Trans}\left(x, a_{i-1}\right)=\left[\begin{array}{cccc}
1 & 0 & 0 & a_{i-1} \\
0 & 1 & 0 & 0 \\
0 & 0 & 1 & 0 \\
0 & 0 & 0 & 1
\end{array}\right] \\
\operatorname{Rot}\left(z, \theta_{i}\right) & =\left[\begin{array}{cccc}
c \theta_{i} & -s \theta_{i} & 0 & 0 \\
s \theta_{i} & c \theta_{i} & 0 & 0 \\
0 & 0 & 1 & 0 \\
0 & 0 & 0 & 1
\end{array}\right], \operatorname{Trans}\left(z, d_{i}\right)=\left[\begin{array}{cccc}
1 & 0 & 0 & 0 \\
0 & 1 & 0 & 0 \\
0 & 0 & 1 & d_{i} \\
0 & 0 & 0 & 1
\end{array}\right]
\end{aligned}
$$

Then:

$$
{ }^{i-1} T_{i}=\left[\begin{array}{cccc}
c \theta_{i} & -s \theta_{i} & 0 & a_{i-1} \\
s \theta_{i} c \alpha_{i-1} & c \theta_{i} c \alpha_{i-1} & -s \alpha_{i-1} & -d_{i} s \alpha_{i-1} \\
s \theta_{i} s \alpha_{i-1} & c \theta_{i} s \alpha_{i-1} & c \alpha_{i-1} & d_{i} c \alpha_{i-1} \\
0 & 0 & 0 & 1
\end{array}\right]
$$

Where $s \theta_{i}=\sin \theta_{i}, c \theta_{i}=\cos \theta_{i}, s \alpha_{i-1}=\sin \alpha_{i-1}, c \alpha_{i-1}=\cos \alpha_{i-1}$.

The parameters of links and joints are calculated in Table 1.

Table 1 The parameters of links and joints

\begin{tabular}{cccccc}
\hline Link & Length & Value & Joint & Angle & Value \\
\hline 1 & $l_{1}$ & $6.5 \mathrm{~cm}$ & 1 & $\beta_{1}$ & $240^{\circ}$ \\
2 & $l_{2}$ & $9.5 \mathrm{~cm}$ & 2 & $\beta_{2}$ & $180^{\circ}$ \\
3 & $l_{3}$ & $8 \mathrm{~cm}$ & 3 & $\beta_{3}$ & $180^{\circ}$ \\
4 & $l_{4}$ & $6 \mathrm{~cm}$ & 4 & $\beta_{4}$ & $180^{\circ}$ \\
5 & $l_{5}$ & $3 \mathrm{~cm}$ & 5 & $\beta_{5}$ & $180^{\circ}$ \\
& & & 6 & $\beta_{6}$ & $90^{\circ}$
\end{tabular}

According to the D-H method, the transformation of links of coordinate system $\{6\}$ relative to $\{0\}$ is: 


$$
{ }^{0} T_{6}={ }^{0} T_{1}^{1} T_{2}^{2} T_{3}^{3} T_{4}^{4} T_{5}^{5} T_{6}=\left[\begin{array}{cccc}
m_{11} & n_{12} & l_{13} & p_{x} \\
m_{21} & n_{22} & l_{23} & p_{y} \\
m_{31} & n_{32} & l_{33} & p_{z} \\
0 & 0 & 0 & 1
\end{array}\right]=\left[\begin{array}{cccc}
\vec{m} & \vec{n} & \vec{l} & \vec{p} \\
0 & 0 & 0 & 1
\end{array}\right]
$$

Where $\vec{m}=\left[\begin{array}{lll}m_{11} & m_{21} & m_{31}\end{array}\right]^{T}, \vec{n}=\left[\begin{array}{lll}n_{12} & n_{22} & n_{32}\end{array}\right]^{T}, \vec{l}=\left[\begin{array}{lll}l_{13} & l_{23} & l_{33}\end{array}\right]^{T}, \vec{p}=\left[\begin{array}{lll}p_{x} & p_{y} & p_{z}\end{array}\right]^{T}$.

Equation (3) is the forward kinematics model of this 6-DOF robotic manipulator, where the vectors $(\vec{m}, \vec{n}, \vec{l})$ represent the direction vectors of the three axes $\left(x_{6}, y_{6}, z_{6}\right)$ of the coordinate system $\{6\}$ in the coordinate system $\{0\}$. The vector $\vec{p}$ represents the position of the origin of coordinates $(H)$ of the coordinate system $\{6\}$ in the coordinate system $\{0\}$. We can figure out the position coordinates of the hand claw relative to the base when we substitute the parameters of links and joints into these equations.

\section{Methodology}

\subsection{System Description}

Considering a robotic manipulator of $\mathrm{N}$-joint, its dynamic performance can be described by a second order nonlinear differential equation ${ }^{[10]}$ :

$$
\boldsymbol{H}(\boldsymbol{q}) \ddot{q}+C(q, \dot{q}) \dot{q}+G(q)+\omega=\tau
$$

Where $q \in R^{n}$ is the vector of joint angular positions, $H(q) \in R^{n \times n}$ is the positive definite inertia matrix, $C(q, \dot{q}) \in R^{n}$ is the vector of centrifugal and Coriolis torque, $G(q) \in R^{n}$ is the vector of gravitational torque, and $\tau \in R^{n}$ is the vector of control torque, $\omega$ is the bounded external disturbance.

The kinetic characteristics of the robot system are as follows:

1) Property 1: $H(q)-2 C(q, \dot{q})$ is a skew symmetric matrix ${ }^{[11]}$;

2) Property 2: The dynamic equation (4) can be rewritten as ${ }^{[12]}$ :

$$
\boldsymbol{H}(q) \dot{q}_{r}+C(q, \dot{q}) q_{r}+G(q)=\Phi\left(q, \dot{q}, q_{r}, \dot{q}_{r}\right) \theta
$$

Where $\Phi\left(q, \dot{q}, q_{r}, \dot{q}_{r}\right)$ is the regressor matrix of known joints variable functions, $q_{r} \in R^{n}, \theta \in R^{n}$ consists of the unknown parameters.

According to [9], make the following assumptions: the norm of external disturbance $\omega$ is satisfied:

$$
\|\omega\| \leq d_{1}+d_{2}\|e\|+d_{3}\|\dot{e}\|
$$

Where $d_{1}, d_{2}, d_{3}$ are respectively the positive constant, $e=q-q_{d}, \dot{e}=\dot{q}-\dot{q}_{d}$ are respectively the tracking error and the derivative of tracking error, $q_{d} \in R^{n}$ is the desired trajectory.

\subsection{The Design of Controller}

Defining the virtual "reference trajectories":

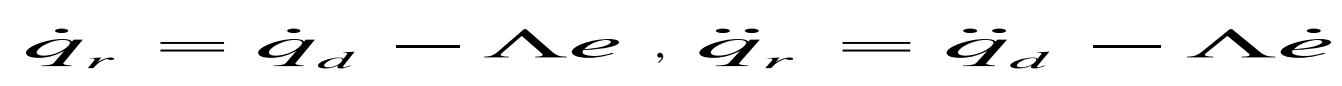


Where $\Lambda=\operatorname{diag}\left(\lambda_{1}, \lambda_{2}, \ldots, \lambda_{n}\right), \lambda_{i}>0, i=1,2, \ldots, n$.

Defining the sliding mode function:

$$
S=\dot{1}=
$$

When the external disturbance is bounded, the control law is:

$$
\begin{aligned}
& \tau=\hat{\boldsymbol{I I}}(q) \dot{q}_{r}+\hat{\mathrm{C}}(q, \dot{q}) \dot{q}_{r}+\hat{G}(q)+u-K_{d} s \\
& \boldsymbol{u}=\left[u_{1}, u_{2}, \ldots, u_{n}\right]^{T}
\end{aligned}
$$

Where $K_{d}=\operatorname{diag}\left(K_{d 1}, K_{d 2}, \ldots, K_{d n}\right), K_{d i}>0, u_{i}=-\left(d_{1}+d_{2}\|e\|+d_{3}\|\dot{e}\|\right) \operatorname{sgn}\left(s_{i}\right)$, $i=1,2, \ldots, n$.

The Lyapunov function is designed that:

$$
V(t)=\frac{1}{2} s^{T} H s+\frac{1}{2} \widetilde{\theta}^{T} \Gamma \widetilde{\theta}
$$

Where $\Gamma=\operatorname{diag}\left(\gamma_{1}, \gamma_{2}, \ldots, \gamma_{n}\right), \gamma_{i}>0, i=1,2, \ldots, n, \hat{\theta}$ is the estimation of $\theta$, which is a constant vector, $\tilde{\theta}=\hat{\theta}-\theta$, so $\dot{\tilde{\theta}}=\dot{\hat{\theta}}$.

Then:

$$
\begin{aligned}
& \dot{V}=s^{T} \boldsymbol{H} \dot{s}+\frac{1}{2} s^{T} \dot{H} s+\widetilde{\theta}^{T} \Gamma \dot{\tilde{\theta}} \\
& =s^{T}\left(\boldsymbol{H} \ddot{\boldsymbol{q}}-\boldsymbol{H} \boldsymbol{M} \ddot{q}_{r}\right)+\frac{1}{2} s^{T} \dot{\boldsymbol{I}} s+\widetilde{\boldsymbol{\theta}}^{T} \boldsymbol{\Gamma} \dot{\tilde{\theta}} \\
& =s^{T}\left(\tau-C \dot{\boldsymbol{q}}-G-\boldsymbol{H} \ddot{q}_{r}-\omega\right)+\frac{1}{2} s^{T} \dot{\boldsymbol{I}} \dot{s}+\widetilde{\boldsymbol{O}}^{T} \boldsymbol{\Gamma} \dot{\tilde{\theta}}
\end{aligned}
$$

Introducing (9) into the above equation:

$$
\begin{aligned}
& \dot{V}=s^{T}\left(\tilde{\boldsymbol{H}} \ddot{q}_{r}+\widetilde{\boldsymbol{C}} \dot{\boldsymbol{q}}_{r}+\widetilde{G}-K_{d} s-C s+u-\omega\right)+\frac{1}{2} s^{T} \dot{H} s+\widetilde{\theta}^{T} \Gamma \dot{\tilde{\theta}} \\
& \text { Where } \widetilde{\boldsymbol{I I}}=\widehat{\boldsymbol{I}}-\boldsymbol{I}, \tilde{\boldsymbol{G}}=\widehat{\boldsymbol{E}}-\bar{C}, \widetilde{\boldsymbol{G}}=\widehat{\boldsymbol{G}}-\boldsymbol{G} \text {. }
\end{aligned}
$$

According to the linear characteristics of robot dynamics equation:

$$
\widetilde{I} \bar{q}_{r}+\tilde{\bar{C}} \dot{q}_{r}+\tilde{G}=\Phi\left(q, q, \dot{q}_{r}, \dot{q}_{r}\right) \tilde{\theta}
$$

Then

$$
\begin{aligned}
\dot{V}= & s^{T}\left(\Phi \tilde{\theta}-K_{d} s-C s+u-\omega\right)+\frac{1}{2} s^{T} \dot{H} s+\widetilde{\theta}^{T} \Gamma \dot{\tilde{\theta}} \\
= & s^{T}\left(\Phi \tilde{\theta}-K_{d} s\right)+s^{T}(u-\omega)+\frac{1}{2} s^{T}(\dot{H}-2 C) s+\widetilde{\theta}^{T} \Gamma \dot{\tilde{\theta}} \\
= & \widetilde{\theta}^{T} \Phi^{T} s-s^{T} K_{d} s+s^{T}(u-\omega)+\widetilde{\theta}^{T} \Gamma \dot{\tilde{\theta}} \\
& \dot{V}=\widetilde{\theta}^{T}\left(\Phi^{T} s+\Gamma \dot{\tilde{\theta}}\right)-s^{T} K_{d} s+s^{T}(u-\omega)
\end{aligned}
$$

The adaptive control law is as follows:

$$
\dot{\hat{\theta}}=-\Gamma^{-1} \Phi^{T}\left(q, q, \dot{q}_{r}, \ddot{q}_{r}\right) s
$$

Then 


$$
\dot{V}=-s^{T} K_{d} s+s^{T}(u-\omega)
$$

And $-s^{T} K_{d} s \leq 0$, so $\dot{V} \leq s^{T}(u-\omega)$.

$$
\begin{aligned}
s^{T} u & =\sum_{i=1}^{n} s_{i}\left[-\left(d_{1}+d_{2}\|e\|+d_{3}\|\dot{e}\|\right) \operatorname{sgn}\left(s_{i}\right)\right] \\
& =\sum_{i=1}^{n}\left[-\left(d_{1}+d_{2}\|e\|+d_{3}\|\dot{e}\|\right)\left|s_{i}\right|\right] \\
& \leq \sum_{i=1}^{n}\left(-\|\omega\| \cdot\left|s_{i}\right|\right) \\
-s^{T} \omega & \leq\left\|s^{T}\right\| \bullet\|\omega\|
\end{aligned}
$$

Where $\left\|S^{T}\right\|=\sum_{i=1}^{n}\left|s_{i}\right|$, then $s^{T}(u-\omega) \leq \sum_{i=1}^{n}\left(-\|\omega\| \cdot \bullet S_{i} \mid\right)+\left\|s^{T}\right\| \bullet\|\omega\|=0$, therefore:

$$
\bar{\gamma} \leq 0
$$

So the position error of robotic manipulator system converge to the sliding mode surface under the effect of control law (9) and (12), then $s=\dot{e}+\Lambda e$ when $t \rightarrow \infty, e \rightarrow 0$, so this algorithm ensures that the steady-state error of joint position is zero and the system is stable.

\section{Simulation}

In order to verify the adaptive algorithm optimized in this paper, we conduct a simulation experiment on a two-link robotic manipulator, and we introduce the traditional PD control algorithm for comparison. The control law of this algorithm is as follows:

$$
\begin{aligned}
& \tau=\hat{\boldsymbol{I}}(q) \ddot{q}_{d}+\hat{\boldsymbol{C}}(q, \dot{q}) \dot{q}_{d}+\hat{\boldsymbol{G}}(q)-K_{p} e-K_{v} \dot{e} \\
& \dot{\hat{\theta}}=-\boldsymbol{\Gamma}^{-1} \Phi^{T}\left(q, q, \dot{q}_{d}, \ddot{q}_{d}\right) \dot{e}
\end{aligned}
$$

Considering a rigid two-link robotic manipulator, the dynamic equation of the robotic manipulator model is given as:

$$
\begin{aligned}
H(q) & =\left[\begin{array}{cc}
p_{1}+p_{2}+2 p_{3} \cos q_{2} & p_{2}+p_{3} \cos q_{2} \\
p_{2}+p_{3} \cos q_{2} & p_{2}
\end{array}\right] \\
C(q, \dot{q}) & =\left[\begin{array}{cc}
-p_{3} \dot{q}_{2} \sin q_{2} & -p_{3}\left(\dot{q}_{1}+\dot{q}_{2}\right) \sin q_{2} \\
p_{3} \dot{q}_{1} \sin q_{2} & 0
\end{array}\right] \\
G(q) & =\left[\begin{array}{c}
p_{1} g_{1} \cos q_{2}+p_{3} g_{1} \cos \left(q_{1}+q_{2}\right) \\
p_{3} g_{1} \cos \left(q_{1}+q_{2}\right)
\end{array}\right]
\end{aligned}
$$

Where $\theta=\left[\begin{array}{lll}p_{1} & p_{2} & p_{3}\end{array}\right]^{T}$ denote the unknown parameters, $g_{1}=g / l_{1}, g$ is the gravity acceleration, $l_{1}$ and $l_{2}$ are the length of link 1 and link $2, g=9.8 \mathrm{~m} / \mathrm{s}^{2}$.

The desired position trajectories are:

The initial states are:

$$
q_{d 1}=q_{d 2}=\cos \left(2 \pi t+\frac{\pi}{2}\right)
$$




$$
q_{1}(0)=0.5, \dot{\boldsymbol{q}}_{1}(\mathbf{O})=\mathbf{O}, q_{2}(0)=0.5, \dot{\boldsymbol{q}}_{2}(\mathbf{O})=\mathbf{O}
$$

The external disturbance is:

$$
d_{1}=1, d_{2}=2, d_{3}=3, \omega=d_{1}+d_{2}\|e\|+d_{3}\|\dot{e}\|
$$

The related parameters of the robotic manipulator are:

$$
l_{1}=1 \mathrm{~m}, l_{2}=0.8 \mathrm{~m}, m_{1}=0.5 \mathrm{~kg}, m_{2}=0.5 \mathrm{~kg}
$$

The control parameters are respectively:

$$
K_{p}=\operatorname{diag}(150,150), K_{v}=\operatorname{diag}(500,500), K_{d}=\operatorname{diag}(150,150), \Lambda=\operatorname{diag}(5,5), \Gamma=\operatorname{diag}(5,5,5)
$$

The expression formula of $\Phi$ is detailed in [10].

Simulation results are shown in the Fig.3 and Fig.4. Fig.3 show the simulation results of the traditional PD algorithm. Fig.4 show the simulation results of the proposed algorithm.

From the Fig. 3 and Fig.4, it can be seen that the new controller can make the position error converge to zero quickly and can compensate the uncertainties caused by the unknown parameters and external disturbances. This algorithm guarantees that the robotic manipulator can track the desired trajectory quickly and effectively. Nevertheless, this algorithm cannot guarantee that the estimated parameters are converge to the true values.
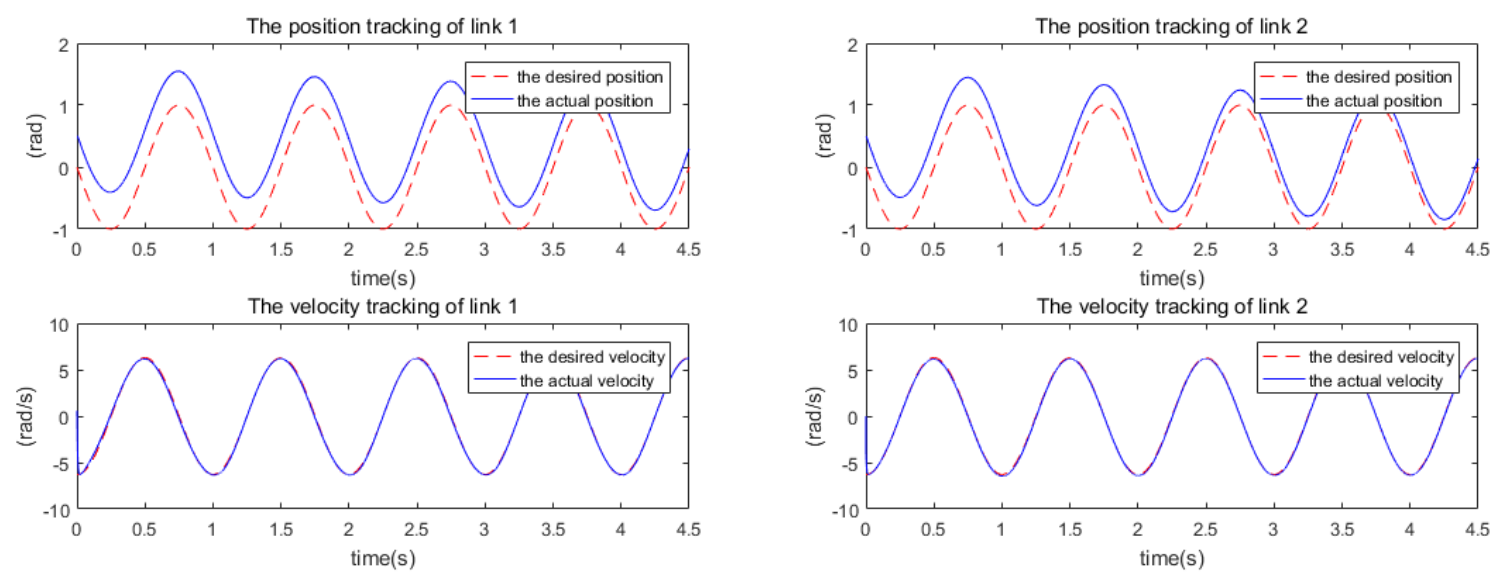

(a) Position tracking and velocity tracking of link 1 (b) Position tracking and velocity tracking of link
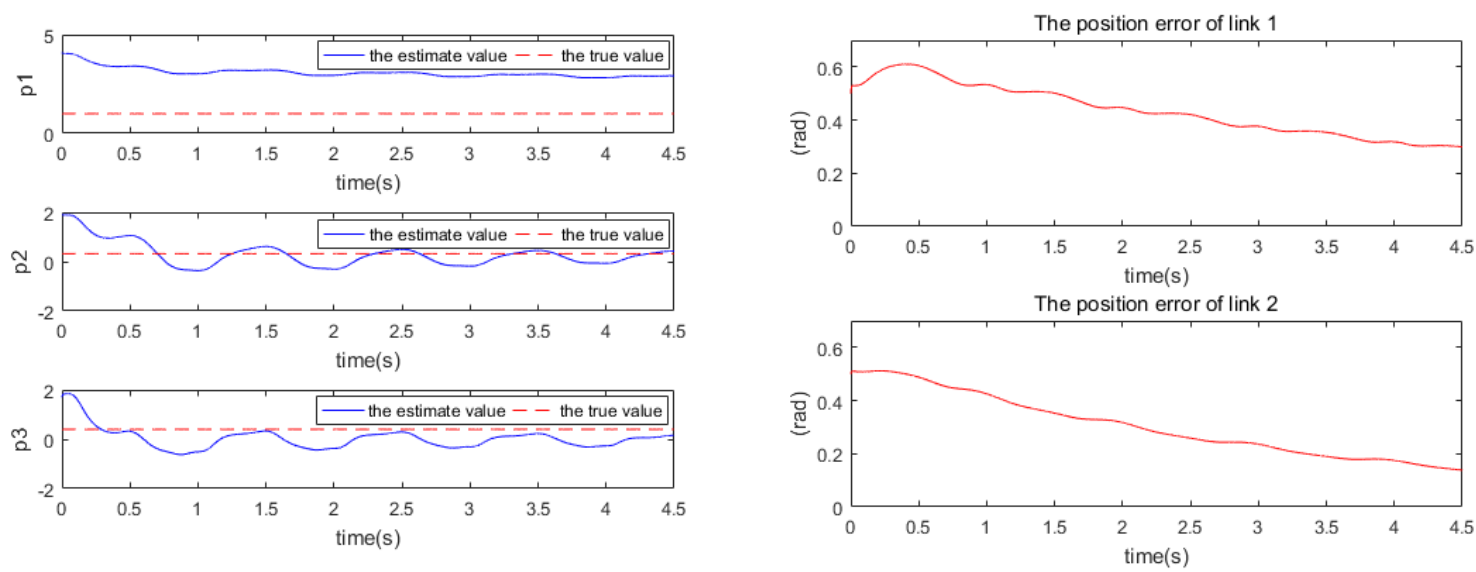

(c) Approximate results of $\theta$

(d) Position error of link 1 and link 2

Fig.3 Simulation results of the PD control algorithm 

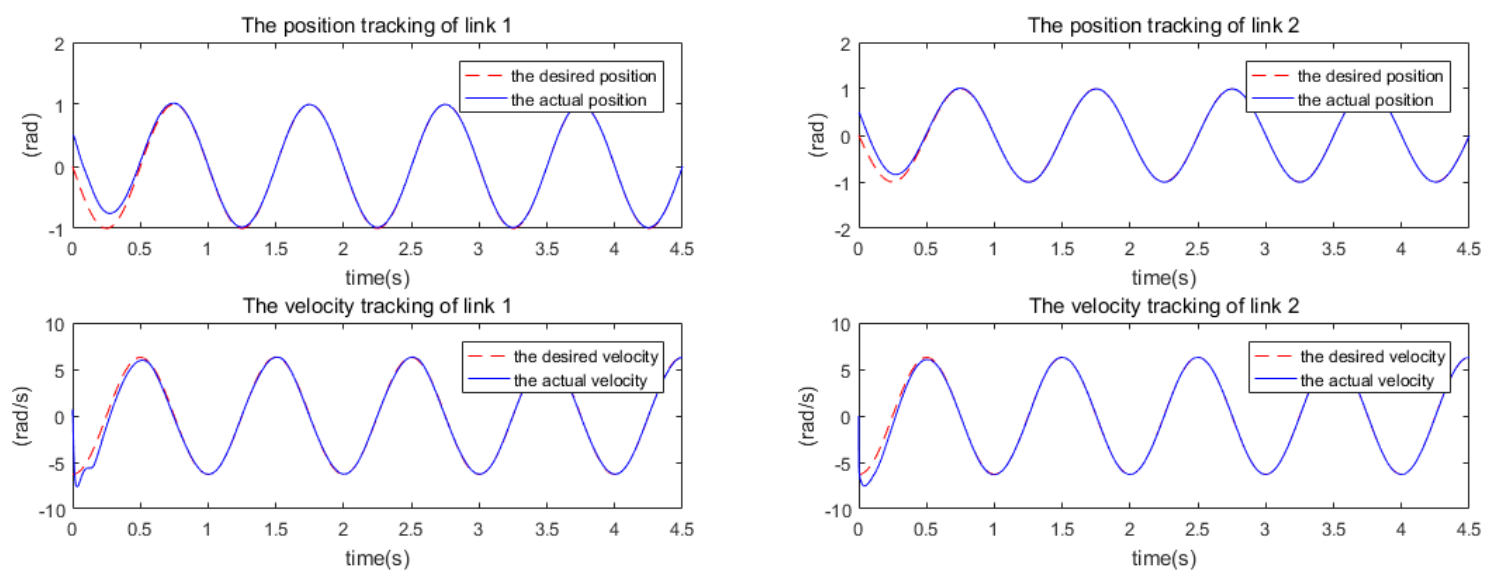

(a) Position tracking and velocity tracking of link 1 (b) Position tracking and velocity tracking of link 2


(c) Approximate results of $\theta$



(d) Position error of link 1 and link 2

Fig.4 Simulation results of the proposed algorithm

\section{Conclusion}

In this paper, we have used D-H method to construct the transformation matrix between each joint of the robotic manipulator, and deduced the positive kinematics model of the robotic manipulator. In view of the poor tracking performance of the 6-DOF series manipulator and the limitations of the conventional adaptive control algorithm, based on the conventional PD control algorithm, we added adaptive algorithm to improve the control performance and trajectory tracking accuracy of the robotic manipulator and designed the sliding mode surface to reduce the system position error. The simulation results showed that this algorithm was effective and feasible. However, this algorithm cannot guarantee that the estimated parameters were converge to the true value. So we will extend our research in this field.

\section{Acknowledgments}

Thanks for the support of the following funds:

1) The National Natural Science Foundation of China. (No.51775325, Bin Ren)

2) Yong Eastern Scholar. (No. QD2016033, Bin Ren)

3) Hong Kong Scholars Program of China. (No. XJ2013015, Bin Ren) 


\section{References}

[1] Liu Jinkun. Sliding Mode Control Design and MATLAB Simulation (Third Edition): The Design Method of Advanced Control System [M]. Beijing: Tsinghua University Press, 2015.

[2] Gao Wei. Research on Sliding Mode Control for Robotic Manipulator Based on RBF Neural Network [D]. Shenyang: Northeastern University, 2017.

[3] MELKOU L, HAMERLAIN M. Classical Sliding and Generalized Variable Structure Controls for a Manipulator Robot Arm with Pneumatic Artificial Muscles [J]. International Journal of System Dynamics Applications (IJSDA), 2014, 3(1).

[4] Luo Shaohua, Wen Zhiling, Lin Zhaoqin. Adaptive Fuzzy SMC Control for Nonlinear System of Dual Arm Robot [J]. Applied Mechanics and Materials, 2012, 2029(224).

[5] Mo Yi. Six degree of freedom series improved adaptive robust control algorithm for robot [J]. Journal of Chinese Agricultural Mechanization, 2016, 37(5): 232-235, 274.

[6] Mu Xiaojiang. Research on Adaptive Neural Sliding Mode Control of Manipulator Robots [A]. Control Engin- eering of China, 2015, 22(4).

[7] AMIN A T M, AB RAHIM A H, Low C Y. Adaptive controller algorithm for 2-DOF humanoid robot arm. Procedia Technology, 2014(15), 765-774.

[8] NEILA M B R, TARAK D. Adaptive Terminal Sliding Mode Control for Rigid Robotic Manipulators [J]. International Journal of Automation \& Computing, 2011, 8(02):215-220.

[9] Xiong Youlun. Fundamentals of Robotics [M]. Wuhan: Hua zhong University of Science and Technology Press, 1996:32-35.

[10] Liu Jinkun. Design of the Robot Control System and MATLAB Simulation [M]. Beijing: Tsinghua University Press, 2008.

[11] Jiao Xiaohong, Li Yunfeng, Fang Yiming, et al. A robust adaptive control method for robot [J]. Robot Technique and Application, 2002, 3:40-43.

[12] Zhao Dongya. Adaptive Full-order Sliding Mode Control of Rigid Robotic Manipulators [A]. Technical Committee on Control Theory, Chinese Association of Automation, Proceedings of the 34th Session of Chinese Control Conference (A) $[C]: 2015,6$.

[13] Li Xiangjun, Yu Xinghuo, Wang Changhong, et al. An adaptive sliding mode control method for mechanical arm system [J]. Journal of System Simulation, 2006, (10): 2895-2897 + 2902.

[14] Wen Jinbei. Kinematics and dynamics analysis of 6-DOF manipulator and robust trajectory tracking control for uncertain rigid robot [D]. Northwestern Polytechnical University, 2002.

[15] Yang Yueneng, Wu Jie, Zheng Wei. Adaptive fuzzy sliding mode control for robotic airship with model uncertainty and external disturbance [J]. Journal of Systems Engineering and Electronics, 2012, 23(2): 250 -255.

[16] Zhang Xu, Zheng Zelong, Qi Yong. Parameter Identification and Calibration of D-H Model for 6-DOF Serial Robots [A]. Robot, 2016, 38(3).

[17] SINGH S, SINGLA E. Modelling and simulation of basic robotic configurations using D-H parameters based adaptive modules [J]. Perspectives in Science, 8(2016): 513-515.

[18] Liu Jinkun. MATLAB Simulation for Sliding Mode Control [M]. Beijing: Tsinghua University Press, 2005.

[19] M.J. Mahmoodabadi, R. Abedzadeh Maafi, M. Taherkhor- sandi. An optimal adaptive robust PID controller subject to fuzzy rules and sliding modes for MIMO uncertain chaotic systems [J]. Applied Soft Computing, 52(2017): 1191-1199. [20] Yu Zhigang, Shen Yongliang, Song Zhongmin. Robust adaptive motion control for manipulator [J]. Control Theory \&Applications, 2011, 28(7).

[21] Tao Hengming. Kinematics Analysis and Control Technology Research of the Six DOF Industrial Robot [D]. Heifei University of Technology, 2014. 